\title{
Autologous and allogeneic ovarian orthotopic transplantation. Morphologic, endocrinologic and natural pregnancy assessment ${ }^{1}$
}

\author{
Luiz Ronaldo Alberti', Leonardo de Souza Vasconcellos" ${ }^{\text {II }}$ Andy PetroianuIII
}

${ }^{\mathrm{I}} \mathrm{PhD}$, Associate Professor, Department of Surgery, Faculty of Medicine, UFMG, Belo Horizonte-MG, Brazil, and Institute of Research, Postgraduate Program of Santa Casa, Belo Horizonte-MG, Brazil. Responsible for acquisition and interpretation of data, statistical analysis, designed the protocol, involved with technical procedures.

"IPhD, Associate Professor, Department of Propedeutics, Faculty of Medicine, UFMG, Belo Horizonte-MG, Brazil. Responsible for acquisition and interpretation of data, statistical analysis, designed the protocol, involved with technical procedures, macroscopic and histopathological examinations, serum hormones evaluation.

IIIPhD, Full Professor, Department of Surgery, Faculty of Medicine, UFMG, Belo Horizonte-MG, Brazil. Responsible for designed the protocol, intellectual and scientific content of the study, supervised all phases of the study, provided guidelines for the surgical interventions.

\section{ABSTRACT}

PURPOSE: To assess pregnancy of rabbits submitted to bilateral ovariectomy and orthotopic allogeneic or autologous intact and sliced ovarian transplantation without a vascular pedicle and to determine the morphofunctional aspects of the transplanted ovaries.

METHODS: Fifty-six female rabbits were studied. The ovaries were removed and orthotopically transplanted or replaced without vascular anastomoses: Group $1(\mathrm{n}=8)$, only laparotomy and laparorrhaphy, Group $2 \mathrm{~A}(\mathrm{n}=8)$ intact ovaries were transplanted on both sides, Group 2B $(n=8)$ both ovaries were sliced and orthotopically transplanted, Group 2C $(n=8)$, an intact ovary was transplanted on one side and a sliced ovary on the other side. In Group 3 the ovaries were reimplanted according to the procedure and subgroups described for Group 2. Three months later, the animals were paired with males for copulation. Estradiol, progesterone, FSH and LH hormone levels were assessed. A histologic study was carried out, and the number of pregnancies and litters were also determined. Chi-square test compared the number of pregnancies and litters. One-way ANOVA and the Tukey-Kramer tests compared the hormonal dosages.

RESULTS: Pregnancies occurred in seven (87.5\%) rabbits of Group 1, in three rabbits (37.5\%) of Groups 2A and 3A, in four rabbits $(50 \%)$ of groups $2 \mathrm{~B}, 3 \mathrm{~B}$ and $3 \mathrm{C}$, and in five $(62.5 \%)$ of group $2 \mathrm{C}$. Normal hormone serum levels and histology confirmed the vitality of all ovaries.

CONCLUSION: Orthotopic allogeneic and autologous ovarian transplantation without a vascular pedicle is viable in rabbits, and preserves their hormonal levels and fertile functions.

Key words: Transplants. Fertility. Ovariectomy. Histology. Pregnancy. Rabbits. 


\section{Introduction}

Most of the experimental studies conducted with ovarian implants have the purpose of maintaining the endocrine function $^{1-3}$. In special situations, when it is necessary to remove the normal ovaries due to cancer or infectious processes of the pelvic organs, the absence of ovarian hormones may provoke various endocrine and functional disorders ${ }^{4,5}$. Among them, the most common are osteoporosis, reduction of libido, sexual dysfunction, and enhancement of lipoprotein levels ${ }^{6}$. In other cases, chemotherapeutic drugs and radiotherapy for the treatment of leukaemias and lymphomas have detrimental effects on the population of ovarian follicles which often result in premature menopause $^{1}$. In addition, developing strategies to manage reproduction in women facing premature menopause would also be useful ${ }^{4,7}$.

The recovery of reproductive capacity has been long neglected by researchers ${ }^{7-10}$. Over the last few years, scientific advances have permitted the occurrence of pregnancy in cases of ovariectomy by means of various methods, such as ovarian cryopreservation, ovarian follicle transplantation, ovarian implants and others ${ }^{11}$.

There are still few reports about the restoration of ovarian function with satisfactory reproductive rate ${ }^{11,12}$. According to several authors, a vascular pedicle is of fundamental importance to preserve the viability of the ovary ${ }^{12,13}$, whereas others have shown that avascular ovary implantation preserves the endocrine function of the organ in rats and mice, but with a low reproductive rate ${ }^{14,15}$.

The aim of the present study was to determine the occurrence of natural pregnancy, as well as the morphologic and endocrine aspects of ovaries after orthotopic allogeneic and autologous ovarian transplantation without vascular anastomosis. In order to define the best ovarian implantation method, intact and sliced transplanted ovaries were compared.

\section{Methods}

This study was performed according to the recommendations of the Guiding Principles in the Care and Use of Animals, and was approved by the Ethics Committee of the Department of Surgery, Medical School, Federal University of Minas Gerais.

Fifty-six sexually mature New Zealand White $(n=28)$ and California $(\mathrm{n}=28)$ female rabbits (Oryctogalus cuniculus) and ten New Zealand White male rabbits with confirmed fertile capacity were used. The animals, ranging in weight from 2450 to $2700 \mathrm{~g}$, were housed in individual cages and received rabbit food and water ad libitum. They were allowed to adapt and were observed for health status for 20 days. Before surgery, the animals were fasted for 12 hours.

The female rabbits were randomly divided into three groups with half of the animals belonging to each breed:

Group $1(\mathrm{n}=8)$, submitted to median laparotomy and laparorrhaphy;

Group 2 ( $n=24)$, submitted to ovariectomy and orthotopic transplantation of both ovaries. These animals were divided into:

- Subgroup 2A ( $\mathrm{n}=8)$ - transplantation of bilateral intact ovaries;

- Subgroup 2B $(n=8)$ - transplantation of bilateral sliced ovaries;

- Subgroup 2C $(n=8)$-transplantation of an intact ovary on one side and of a sliced ovary on the other side.

Group $3(\mathrm{n}=24)$, submitted to ovariectomy and orthotopic reimplantation of both ovaries. These animals were divided into:

- Subgroup 3A $(n=8)$ - reimplantation of bilateral intact ovaries;

- Subgroup 3B $(n=8)$ - reimplantation of bilateral sliced ovaries;

- Subgroup 3C $(n=8)$ - reimplantation of an intact ovary on one side and of a sliced ovary on the other;

All procedures were carried out under aseptic conditions in the Laboratory of Experimental Surgery of the Department of Surgery of the Medical School of the Federal University of Minas Gerais.

One hour before surgery, all animals received $50 \mathrm{mg} / \mathrm{kg}$ of cefadroxil (Cefamox; Bristol-Myers-Squibb, Sao Paulo, Brazil) dissolved in $10 \mathrm{ml}$ of milk and administered through a 12-French orogastric tube. The animals undergoing transplantation also received $50.0 \mathrm{mg} / \mathrm{kg}$ of cyclosporine (Sigmasporin; Nature PlusSigma Pharma, Sao Paulo, Brazil). Anesthesia was obtained with an intramuscular injection of $50 \mathrm{mg} / \mathrm{kg}$ ketamine hydrochloride (Ketalar, Pfizer, Sao Paulo, Brazil). After shaving the abdomen, antisepsis was obtained with a PVPI solution followed by a $2 \%$ alcoholic solution of iodine.

The animals of group 1 were submitted to infraumbilical median laparotomy, identification of the uterus, tubes and ovaries, followed by laparorrhaphy in two continuous layers using 2-0 polyglactine (Vicryl) and 3-0 nylon sutures.

Each animal submitted to transplantation was weighed weekly to adjust the cyclosporine dose. Immunosuppression with a daily cyclosporine solution was obtained during the nine-month research period $(50.0 \mathrm{mg} / \mathrm{kg}$ of body weight). During the first 
three days after surgery, all rabbits received the cefadroxil solution through the orogastric tube.

In the group 2, a pair of female rabbits - a White New Zealand and a California - were operated simultaneously. The animals were submitted to bilateral ovariectomy with special care not to damage the integrity of the Fallopian tubes. Both ovaries were orthotopically changed between the two breeds of rabbits so that each animal was donor and at the same time receiver, according to the subgroup and sutured with 5-0 nylon. The ovaries were sutured close to the Fallopian fimbriae without touching the tubes. Subgroup 2A ( $\mathrm{n}=8)$ was submitted to bilateral transplantation of intact ovaries and sutured with 5-0 nylon. In subgroup 2B $(n=8)$ both ovaries were longitudinally cut into three $2 \mathrm{~mm}$-thick slices and orthotopically sutured with 5-0 nylon. In subgroup $2 \mathrm{C}(\mathrm{n}=8)$, after removal, one ovary was cut in three slices, the other was left intact and both were transplanted. The whole surgical time ranged between 12 and 16 minutes. All ovarian transplants were done without vascular rebuilding. The whole surgical time ranged between 12 and 16 minutes.

The rabbits of group 3 had their ovaries reimplanted according to the subgroups using the same procedure described for Group 2 with the only difference of not changing the ovaries between the two breeds.

After the surgical procedure and during the follow-up period, the female rabbits received food and filtered water ad libitum in separate containers and were maintained in individual cages.

After a three-month-period after the surgical procedures, the females of the transplanted, reimplanted and control groups were daily paired with a fertile males from a different breed of the females ones. The males were randomly changed among the cages with the female rabbits of all groups. After getting pregnant the females were maintained isolated in their cages until the end of the pregnancy and they were not paired again. The number of successful pregnancies and litters born of each rabbit, and the gestational complications were registered.

Nine month after the surgical procedure, serum levels of estradiol, progesterone, follicle stimulating hormone, and luteining hormone were assessed. The reference values of these hormones were based on the results obtained from animals of Group 1, known to be fertile, healthy and of a similar age to that of the rabbits submitted to allogeneic and autologous transplantation. The hormones were quantified by radioimmunoassay and immunofluorimetry.

At the end of the nine-month-follow-up period, all female rabbits were killed by anesthesia with ketamine $(50 \mathrm{mg} / \mathrm{kg})$ and excess ether inhalation The uterus, both ovaries and the Fallopian tubes were removed for morphologic study. The samples were fixed in Bouin's solution and prepared as paraffin-embedded wax blocks. Serial sections were cut at a thickness of $5 \mu \mathrm{m}$ and stained with hematoxylin and eosin for routine histologic examination.

The chi-square test with Yates correction was used to compare the number of successful pregnancies in each group, oneway ANOVA and the Tukey-Kramer tests were used to compare the number of litters for each rabbit and the hormone levels of the groups. The differences were considered significant for $\mathrm{p}<0.05$.

\section{Results}

All the animals recovered spontaneously from surgery, had an uneventful follow-up and survived during the nine months of this experiment period. The weight of the rabbits increased uniformly and no overweight animal was registered.

Table 1 shows the hormone levels of all groups. Ovarian hormones were detected in all the rabbits with allogeneic and autologous transplanted ovaries, even in the animals that not became pregnant. No difference was observed in the hormone levels between the groups and subgroups of rabbits in comparison to the control-group, except for subgroup $2 \mathrm{C}$ who presented higher level of estradiol $(\mathrm{p}<0.05)$

TABLE 1 - Hormone levels (mean and standard deviation) and successful pregnancies in control rabbits and in the rabbits submitted to allogeneic and autologous orthotopic ovarian transplantation.

\begin{tabular}{ccccc}
\hline GROUPS & $\begin{array}{c}\text { ESTRADIOL } \\
(\mathbf{p g} / \mathbf{m l})\end{array}$ & $\begin{array}{c}\text { PROGESTERONE } \\
(\mathbf{p g} / \mathbf{m l})\end{array}$ & $\begin{array}{c}\text { FSH } \\
(\mathbf{U I} / \mathbf{l})\end{array}$ & $\begin{array}{c}\text { LH } \\
(\mathbf{U I} / \mathbf{l})\end{array}$ \\
\hline 1 & $2.801 \pm 762$ & $104.200 \pm 2.348$ & $0.13 \pm 0.05$ & $0.16 \pm 0.07$ \\
2A & $4.063 \pm 927$ & $102.906 \pm 962$ & $0.13 \pm 0.04$ & $0.18 \pm 0.07$ \\
2B & $3.844 \pm 821$ & $103.172 \pm 1.938$ & $0.14 \pm 0.07$ & $0.18 \pm 0.07$ \\
2C & $4.510 \pm 1.246^{*}$ & $103.713 \pm 2.413$ & $0.13 \pm 0.04$ & $0.16 \pm 0.07$ \\
3A & $2.500 \pm 950$ & $109.300 \pm 5500$ & $0.30 \pm 0.20$ & $0.30 \pm 0.20$ \\
3B & $2.850 \pm 700$ & $105.400 \pm 3500$ & $0.20 \pm 0.10$ & $0.20 \pm 0.10$ \\
3C & $2.950 \pm 850$ & $106.100 \pm 3300$ & $0.30 \pm 0.20$ & $0.20 \pm 0.10$ \\
One way & $\mathrm{p}=0.0084$ & $\mathrm{p}=0.9056$ & $\mathrm{p}=0.6455$ & $\mathrm{p}=0.7363$ \\
ANOVA & & & & $\mathrm{p}>0.05$ \\
Tukey-Kramer & $\mathrm{p}<0.05$ & $\mathrm{p}>0.05$ & & \\
test & & & & \\
\hline
\end{tabular}

*Difference between subgroup $2 \mathrm{C}$ and the other subgroups, significant for $\mathrm{p}<0.05$ (Tukey-Kramer test)

G1 - Control group - G2A - Transplantation of intact ovaries - G2B - Transplantation of sliced ovaries - G2C - Transplantation of intact and sliced ovaries - G3A - Reimplantation of intact ovaries - G3B Reimplantation of sliced ovaries - G3C - Reimplantation of intact and sliced ovaries - FSH - Follicle stimulating hormone - LH - Luteinizing hormone 
At the second laparotomy, the abdominal cavity of the rabbits of group 1 was normal. The macroscopic aspect of the reproductive organs of the animals was well preserved without inflammatory signs. There were adhesions between the tubes and ovaries in two rabbits. The uterus and tubes did not show anatomic abnormalities.

At the time of second laparotomy, the macroscopic aspect of the reproductive organs of the animals of groups 2 and 3 was close to normal with little fibrosis and abdominal adhesions. The uterus and tubes did not show anatomic abnormalities and angiogenesis were observed on several ovaries surface. In three rabbits of group 2 and in two animals of group 3 with no pregnancy, adhesions were observed between the tubes and ovaries. In these cases, the ovaries were surrounded by the omentum, the small and large bowel.

Histologic examination confirmed the preservation of ovarian structure. No differences were found between intact and sliced allogeneic and autologous transplanted ovaries. A welldeveloped vascular net formed by angiogenesis surrounded all transplanted ovaries. Many follicles in different phases of maturation and pregnancies indicated the recovery of reproductive function of the ovaries in all groups (Figure 1).

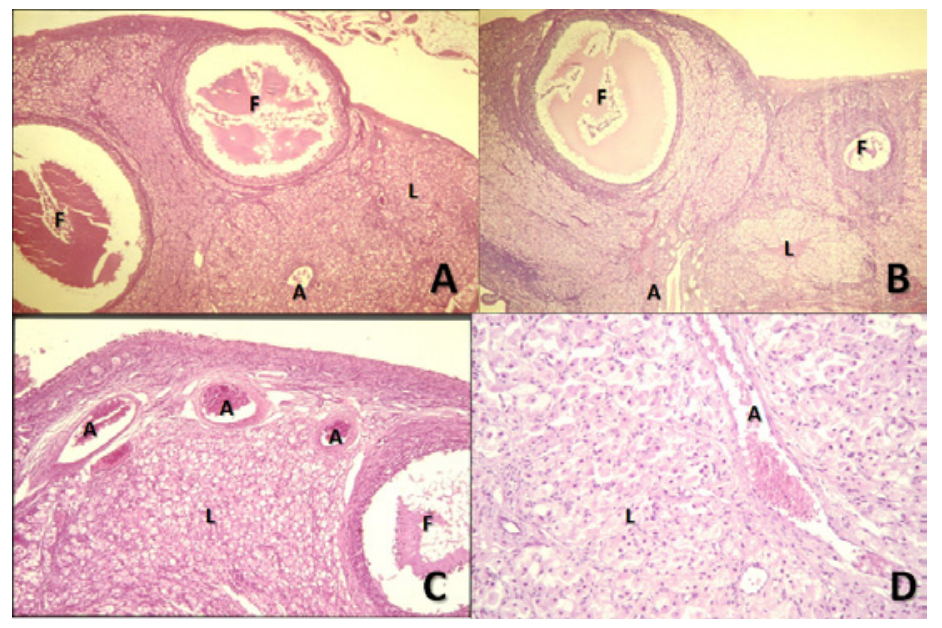

FIGURE 1 - Histological findings on autologous and allogeneic transplanted ovarium without a vascular pedicle. No sign of ischaemia or necrosis is present. Observe follicle of Graaf (F), angiogenesis (A) and corpus luteum (L). Haematoxylin and eosin stain. A - Intact reimplanted ovarium (100x), B - Sliced reimplanted ovarium (100x), C - Intact transplanted ovarium (100x) and D - Sliced transplanted ovarium (400x).

The Fallopian tubes of the rabbits that became pregnant presented a normal aspect, with well-developed microvilli and lack of inflammatory or degenerative signs. In contrast, among the infertile rabbits, two animals of group 2 and other two of group 3 showed salpingitis and two rabbits of Group 2 showed multiple local adhesions among ovaries, tubes and small bowel. The uterus of all animals presented a normal aspect. The rest of the abdominal cavity remained normal, without gross alterations of the other abdominal organs.

The uterine histoarchitecture of all rabbits was preserved. The endometrium showed various proliferation phases compatible with the phases of the estrous cycle, indicating an appropriate hormone production.

Seven (87.5\%) rabbits of Group 1 maintained pregnancy until the end. Parturitions occurred during the fourth month after operation in four rabbits, and during the sixth month after operation in the other three animals. The numbers of animals varied between six to ten litters.

Three of the rabbits of subgroup 2A (37.5\%) maintained pregnancy until the end. Parturitions occurred during the fourth month after operation in one rabbit with the birth of two litters, in the fifth month in another with the birth of three litters, and in another during the sixth month after operation with the birth of one litter.

Of the eight rabbits of subgroup 2B, four (50\%) maintained pregnancy until the end. Birth occurred during the fifth postoperative month in one rabbit, with the birth of three litters; during the sixth month with the birth of two litters; and during the seventh postoperative month in the last two, with one litters in one and two in the other.

Of the eight rabbits of subgroup 2C, five (62.5\%) maintained pregnancy until the end. Parturition occurred during the fifth postoperative month in two rabbits, two months after the beginning of copulation, with the birth of one litter; during the sixth postoperative month in another, with the birth of three litters; and during the eighth postoperative month in the other two, one with one litter and the other with two litters. There were no difference in the time between the beginning of the sexual intercourse and the birth of the nestlings in relation to the two studied female breeds.

Three of the rabbits of subgroup 3A (37.5\%) maintained pregnancy until the end. Parturitions occurred during the fifth month after operation in two rabbits, with the birth of two and three litters, respectively, and during the sixth month after operation with the birth of one litter in another.

Of the eight rabbits of subgroup 3B, four (50\%) maintained pregnancy until the end. Birth occurred during the fifth postoperative month in one rabbit, with the birth of four litters; during the sixth month with the birth of one litter in another; and during the seventh postoperative month in the last two, with two litters in one and three in the other.

Of the eight rabbits of subgroup 3C, four (50\%) maintained pregnancy until the end. Parturition occurred during 
the fifth postoperative month in one rabbit, two months after the beginning of copulation, with the birth of one litter; during the sixth postoperative month in another, with the birth of three litters; and during the eighth postoperative month in the other two, one with one litter and the other with two litters. There were not differences among the control-group and the other groups in relation to the number of gestations (Table 2).

TABLE 2 - Number of successful pregnancies and the number of litters (mean and standard deviation) for each rabbit for all groups.

\begin{tabular}{|c|c|c|c|}
\hline \multirow{2}{*}{$\begin{array}{c}\text { GROUPS } \\
\qquad(\mathrm{N}=8)\end{array}$} & \multicolumn{2}{|c|}{ PREGNANT RABBITS } & \multirow{2}{*}{$\begin{array}{l}\text { NUMBER OF LITTERS } \\
\text { FOR EACH RABBIT } \\
(\text { mean } \pm \text { SD })\end{array}$} \\
\hline & $\begin{array}{c}\text { Number } \\
\text { and } \%\end{array}$ & $\begin{array}{l}\text { chi-square } \\
\text { (p value) } *\end{array}$ & \\
\hline 1 & $7(87.5)$ & - & $6.7 \pm 2.5 * *$ \\
\hline $2 \mathrm{~A}$ & $3(37.5)$ & 0.121 & $2.0 \pm 1.0$ \\
\hline $2 \mathrm{~B}$ & $4(50.0)$ & 0.280 & $2.0 \pm 0.8$ \\
\hline $2 \mathrm{C}$ & $5(62.5)$ & 0.563 & $1.6 \pm 0.9$ \\
\hline $3 \mathrm{~A}$ & $3(37.5)$ & 0.121 & $2.0 \pm 1.0$ \\
\hline $3 \mathrm{~B}$ & $4(50.0)$ & 0.280 & $2.5 \pm 1.3$ \\
\hline $3 \mathrm{C}$ & $4(50.0)$ & 0.280 & $1.7 \pm 0.9$ \\
\hline
\end{tabular}

*Comparison between $\mathrm{G} 1$ and the others groups

**Difference between Group 1 and the other subgroups, significant for $\mathrm{p}$ $<0.001$ (Tukey-Kramer test)

G1 - Control group - G2A - Transplantation of intact ovaries - G2B - Transplantation of sliced ovaries - G2C - Transplantation of intact and sliced ovaries - G3A - Reimplantation of intact ovaries - G3B Reimplantation of sliced ovaries - G3C - Reimplantation of intact and sliced ovaries

\section{Discussion}

The aim of autologous and allogeneic ovarian transplantation is not only to preserve the hormonal function of the organs and to prevent the adverse effects of perimenopausal period $^{6}$, but also to restore reproductive capacity ${ }^{8,12}$.

Experimental studies on ovarian autoimplants have been performed since the XVIII century, but this procedure became relevant only in recent years ${ }^{11}$. The multiplicity of animal species and methods of investigating the ovarian function is responsible for the controversial informations of the literature ${ }^{4,13,15}$. The choice for rabbit was due to its intense reproductive capacity and feasibility of the surgical procedure without special instruments ${ }^{16,17}$.
The ketamine was used by its characteristics of inducing mitigation state, immobility and accentuated analgesia with wide margin of safety and easy control of the anesthetic level.

The cyclosporine (CyA) was given only to females of Group 2 (A, B and C). The animals of Group 1 (control) were not immune suppressed because they have not been submitted to transplant. It was previously demonstrated that CyA interferes with the endocrine system by enhancing the serum level of estradiol. In this regard, alterations of gonadal function was verified in rats and in humans. Interaction between CyA and prolactin on the same lymphocyte receptor has been reported as a mechanism of action of $\mathrm{CyA}^{21}$. Thus, the use of cyclosporine might be of influence on the number of litters by a possible adverse role of this drug, reducing the female fertility or the number of viable litters. Based on this theory, the number of litters obtained after the ovarian transplantation in the present study was lower than is usually verified in normal animals. However, the small amount of rabbits used in each group was probably responsible for the non significant difference between Groups 1 and 2 .

The number of litters obtained after the allogeneic and autologous ovarian transplantation in the present study was lower than usually verified in normal animals. However, due to the little amount of animals in each group, this difference was not significant.

The microscopic study did not show evidences to suggest a hyper or hypo-hormonal function, as for instance, endometrial hypotrophy or hyperplasia. This fact was also proved by the estradiol and progesterone dosages that were normal. There was no difference between the time to become pregnant of the studied groups and normal rabbits in captivity.

The orthotopic ovarian transplanted without surgical revascularization recovered the hormonal and reproductive function. Thus the absence of vascular anastomosis does not seem to interfere with the viability and function of the ovaries, which developed sufficient vascular neoformation. Transplanted ovaries without a vascular anastomosis in rabbits are viable and preserve their endocrine function without detectable adverse effects. These findings have been verified previously in mice ${ }^{15-17}$, and differ from those published by authors who consider the surgical revascularization fundamental to maintain ovarian function ${ }^{12,13}$. Avascular ovarian transplantation avoids the microsurgery that is required to perform difficult arterial and venous anastomoses in small animals. This procedure would also increase the operation time with more risks for the animal. However it is important to acknowledge that long-term survival of ovaries transplanted with and without a vascular pedicle needs to be determined. 
The neovascularization developed around the ovaries is sufficient to preserve their vitality ${ }^{16}$. According to literature, the ovarian tissue is a rich source of angiogenic factors that stimulate a rapid migration of endothelial cells in cases of transplantations, leading to the reestablishment of the blood flow ${ }^{12,15}$. This phenomenon occurs even when the ovaries are implanted in other sites ${ }^{15-17}$. Dissen et al. ${ }^{18}$ implanted ovarian cortex near the jugular vein, and verified the revascularization of the graft after 48 hours, with an increasing expression of specific angiogenic factors, mainly the endothelial growth factor. According to these authors, the gonadotropin secretion plays a pivotal role in the angiogenesis. Among the gonadotropins, FSH seems to be the most important, due to the stimulation of cellular mitosis and inhibition of apoptosis of the granular layer. The treatment of antral mice follicles with growing doses of FSH causes a suppression of DNA fragmentation, reaching the maximum suppression of $60 \%{ }^{4}$. Probably, due to the privation of mitogenic and trophic factors, as the gonadotropins and the ovarian steroids, apoptosis is observed in ovarian cells ${ }^{18}$. The concentration of FSH was maintained inside the normality limits in the studied groups.

All animals recovered their ovarian cyclicity indicating both procedures lead to a vital implant. Thus the endocrine recoverance is complete enough to establish normal estral cycles, resulting in pregnancy. The absence of surgical reestablishment of vascularization did not interfere with normal ovarian function in both sliced or integer ovaries.

Two different methods of ovarian transplantation (intact or sliced) were compared in order to assess the influence of the contact surface on the restoration of the ovarian function. Previous studies $^{15-17}$ have shown that sliced transplanted ovaries are more effective in promoting normal estrous cycles than intact ones, due to a greater contact surface with the blood supply. However, in the present investigation no difference was observed in the histoarchitecture of the ovaries.

Although the fertility rate of subgroups $2 \mathrm{~A}$ and $3 \mathrm{~A}$ was lower than subgroups $2 \mathrm{~B}, 2 \mathrm{C}, 3 \mathrm{~B}$ and $3 \mathrm{C}$, these results were not significantly different, probably due to the little amount of animals in each group. It is worth to emphasize that the percentage of litters obtained in the present study was higher than the data of the literature ${ }^{12,13}$

The absence of pregnancy in half of the rabbits of groups 2 and 3 may have been due, at least in part, to adhesions surrounding the tubes, and probably preventing fertilization. We could not find the factors related to those adhesions other than natural inflammatory reaction of organism to trauma. The possibility of post-transplant hypoxia in the ovaries because of a lack of vascular pedicle should be considered. However, the adhesions were not exclusively found around the ovaries, but throughout the abdomen. Although pregnancy was not detected, the endocrine function of these rabbits was preserved. Undetected pregnancies may have occurred in both groups, since abortions or other abnormalities of pregnancy could not be assessed.

\section{Conclusion}

Intact or sliced orthotopic allogeneic and autologous ovarian transplantation without a vascular pedicle is viable in rabbits and these animals may conceive and deliver litters naturally.

\section{References}

1. Fish JD. Fertility preservation for adolescent women with cancer. Adolesc Med State Art Rev. 2012;23:111-22.

2. Grynberg M, Poulain M, Sebag-Peyrelevade S, le Parco S, Fanchin R, Frydman N. Ovarian tissue and follicle transplantation as an option for fertility preservation. Fertil Steril. 2012;97:1260-8.

3. Suzuki N, Hashimoto S, Igarashi S, Takae S, Yamanaka M, Yamochi T, Takenoshita M, Hosoi Y, Morimoto Y, Ishizuka B. Assessment of long-term function of heterotopic transplants of vitrified ovarian tissue in cynomolgus monkeys. Hum Reprod. 2012;27:2420-9.

4. Callejo J, Jauregui MT, Valls C. Heterotopic ovarian transplantation without vascular pedicle in syngeneic Lewis rats. Fertil Steril. 1999;72:513-7.

5. Wang Y, Chang Q, Sun J, Dang L, Ma W, Hei C, Shen X, Zhao C, Cai Y, Pei X, Zhang X, Wang Y, Jiang X. Effects of HMG on revascularization and follicular survival in heterotopic autotransplants of mouse ovarian tissue. Reprod Biomed Online. 2012;24:646-53.

6. Calero JA, Curiel MD, Moro MJ, Carrascal MT, Santana JS, Avial MR. Speed of sound, bone mineral density and bone strength in oophorectomized rats. Eur J Clin Invest. 2000;30:210-4.

7. Commin L, Buff S, Rosset E, Galet C, Allard A, Bruyere P, Joly T, Guérin P, Neto V. Follicle development in cryopreserved bitch ovarian tissue grafted to immunodeficient mouse. Reprod Fertil Dev. 2012;24(3):461-71.

8. Nisolle M, Casanas-Roux F, Qu J, Motta P, Donnez J. Histologic and ultrastructural evaluation of fresh and frozen-thawed human ovarian xenografts in nude mice. J Fertil Steril. 2000;74:122-9.

9. Milenkovic M, Diaz-Garcia C, Wallin A, Brännström M. Viability and function of the cryopreserved whole rat ovary: comparison between slow-freezing and vitrification. Fertil Steril. 2012;97:117682.

10. Damous LL, Silva SM, Lopes RA, Sakano CR, Simões M de J, Montero EF. Study on the vaginal smear of rats submitted to autologous ovarian transplant: impact of remote ischemic preconditioning. Acta Cir Bras. 2009;24:387-92.

11. Nugent D, Meirow D, Brook PF, Aubard Y, Gosden RG. Transplantation in reproductive medicine. Hum Reprod Update.1997;3:267-80.

12. Green CJ, Simpkin S, Grimaldi G. Pregnancy after autografting and allografting vascularized ovaries and en bloc vascularized ovaries with adnexa in rabbits. Br J Obstet Gynaecol. 1982;89:645-51.

13. Wang X, Bilolo KK, Qi S, Xu D, Jiang W, Vu MD, Chen H. Restoration of fertility in oophorectomized rats after tubo-ovarian 
transplantation. Microsurgery. 2002;22:30-3.

14. Gutierrez GA, Vargas Aguirre MA, Corona Martha A, Becerra E, Gonzalez Ortega C, Mendoza Hurtado S, Monroy Avendano E, Tovar Caballero G. Gestation in sheep after autotransplantation with cryopreserved ovarian tissue: evidence for oocyte viability after the freezing and transplantation procedures. Gynecol Obstet Mex. 2003;71:5-11.

15. Alberti LR, Vasconcellos LS, Petroianu A, Nunes MB. Avaliação morfofuncional do auto-implante ovariano no retroperitônio. Medicina (Ribeirão Preto). 2002;35:30-5.

16. Alberti LR, Vasconcellos LS, Barbosa JF, Petroianu A. Implante autólogo ovariano no omento maior. Rev Bras Ginecol Obstet. 2002;24:187-92.

17. Petroianu A, Vasconcellos LS, Alberti LR, Castro LPF, Leite JM. Natural pregnancy in rabbit that underwent oophorectomy and orthotopic allogeneic or autologous ovarian transplantation. Fertil Steril. 2002;77:1298-9.

18. Dissen GA, Lara HE, Fahrenbach WH, Costa ME, Ojeda SR. Immature rat ovaries become revascularized rapidly after autotransplantation and show gonadotropin-dependent increase in angiogenic factor gene expression. Endocrinology. 1994;134:114654.

\section{Correspondence:}

Prof. Luiz Ronaldo Alberti

Rua Professor Baroni, 151/401

30441-180 Belo Horizonte - MG Brasil

Tel.: (55-31)3504-1576

luizronaldoa@yahoo.com.br

Received: August 19, 2012

Review: October 21, 2012

Accepted: November 22, 2012

Conflict of interest: none

Financial sources: CNPq and FAPEMIG

${ }^{1}$ Research performed at Laboratory of Experimental Surgery, Department of Surgery, Medicine School, Federal University of Minas Gerais (UFMG), Belo Horizonte-MG, Brazil. 